\title{
Families of vector-like deformations of relativistic quantum phase spaces, twists and symmetries
}

\author{
Daniel Meljanac ${ }^{1, \mathrm{a}}$, Stjepan Meljanac ${ }^{2, \mathrm{~b}}$, Danijel Pikutić ${ }^{2, \mathrm{c}}$ \\ ${ }^{1}$ Division of Materials Physics, Ruđer Bošković Institute, Bijenička c.54, 10002 Zagreb, Croatia \\ ${ }^{2}$ Division of Theoretical Physics, Ruđer Bošković Institute, Bijenička c.54, 10002 Zagreb, Croatia
}

Received: 15 September 2017 / Accepted: 6 November 2017 / Published online: 6 December 2017

(C) The Author(s) 2017. This article is an open access publication

\begin{abstract}
Families of vector-like deformed relativistic quantum phase spaces and corresponding realizations are analyzed. A method for a general construction of the star product is presented. The corresponding twist, expressed in terms of phase space coordinates, in the Hopf algebroid sense is presented. General linear realizations are considered and corresponding twists, in terms of momenta and Poincaré-Weyl generators or $\mathfrak{g l}(n)$ generators are constructed and R-matrix is discussed. A classification of linear realizations leading to vector-like deformed phase spaces is given. There are three types of spaces: (i) commutative spaces, (ii) $\kappa$-Minkowski spaces and (iii) $\kappa$-Snyder spaces. The corresponding star products are (i) associative and commutative (but non-local), (ii) associative and noncommutative and (iii) non-associative and non-commutative, respectively. Twisted symmetry algebras are considered. Transposed twists and left-right dual algebras are presented. Finally, some physical applications are discussed.
\end{abstract}

\section{Introduction}

Reconciliation of quantum mechanics and general relativity, leading to a formulation of quantum gravity, is a longstanding problem in theoretical physics. At very high energies, gravitational effects can no longer be neglected and spacetime is no longer a smooth manifold but rather a fuzzy space or some type of non-commutative space $[1,2]$. Noncommutative geometry is one of the candidates for describing the physics at the Planck scale. Combined analyses of Einstein's general relativity and Heisenberg's uncertainty principle lead to a very general class of non-commutative spacetimes [1], for example Gronenwald-Moyal plane [3,4]

\footnotetext{
a e-mail: Daniel.Meljanac@irb.hr

be-mail: meljanac@irb.hr

c e-mail: dpikutic@irb.hr
}

and $\kappa$-Minkowski algebra [5-11]. Generally, physical theories on non-commutative manifolds require a new framework of non-commutative geometry [12]. In this framework, the search for generalized (quantum) symmetries that leave the physical action invariant leads to deformation of the Poincaré symmetry, with $\kappa$-Poincaré symmetry being one of the ones most extensively studied [5-10,13-22]

One example of a deformed relativistic symmetry that could describe the physics at the Planck scale is the $\kappa$ deformed Poincaré Hopf algebra symmetry, where $\kappa$ is the deformation parameter, usually corresponding to the Planck scale. It has been shown that a quantum field theory with $\kappa$-Poincaré symmetry emerges in a certain limit of quantum gravity coupled to matter fields [23-27], which amounts to a non-commutative field theory on the $\kappa$-deformed Minkowski space.

It is well known $[3,28-30]$ that deformations of a symmetry group can be realized through application of Drinfeld twists on that symmetry group [31-34]. The main virtue of the twist formulation is that the deformed, twisted symmetry algebra is the same as the original undeformed one. There is only a change in the coalgebra structure [3], which then leads to the same single particle Hilbert space and free field structure as in the corresponding commutative theory.

In [35], complete analysis of linear realizations for $\kappa$ Minkowski space that are expressed in terms of generators of the $\mathfrak{g l}(n)$ algebra was given. A method for constructing Drinfeld twist operators, corresponding to each linear realization of $\kappa$-Minkowski space satisfying cocycle and normalization condition was presented. Symmetries generated by Drinfeld twists were classified and $\kappa$-Minkowski space was embedded into the Heisenberg algebra having natural Hopf algebroid structure. In [36], a method for the construction of the star product and twist in Hopf algebroid sense was presented.

Assuming vector-like deformations, we aim to explore which kinds of deformed relativistic quantum phase spaces can arise. In the present paper we consider general vector- 
like deformations of relativistic quantum phase space and expand results from [35] to all possible linear realizations and we construct the corresponding twists. Linear realizations and vector-like deformed spaces are classified in three types and the related symmetry algebras are presented.

In Sect. 2, general vector-like deformed relativistic quantum phase spaces are constructed and corresponding realizations are presented. In Sect. 3, the method for general construction of star product and twist (expressed in terms of phase space coordinates) in Hopf algebroid sense is presented. In Sect. 4, linear realizations are considered and corresponding twists (expressed in terms of momenta and Poincaré-Weyl generators or $\mathfrak{g l}(n)$ generators) are constructed. Also, the R-matrix is discussed. In Sect. 5, a classification of linear realizations leading to vector-like deformed phase spaces is given. There are three types of spaces: (i) commutative spaces, (ii) $\kappa$-Minkowski spaces and (iii) $\kappa$ Snyder spaces. In Sect. 6, twisted symmetry algebras are considered. In Sect. 7, transposed twists and left-right dual algebras are presented. Finally, an outlook and a discussion are given in Sect. 8.

\section{Vector-like deformations of relativistic quantum phase spaces and realizations}

Let us start with deformed phase space (deformed Heisenberg algebra) $\hat{\mathcal{H}}$ generated by commutative momenta $p_{\mu}$ and generically non-commutative coordinates $\hat{x}_{\mu}, \mu=$ $0,1, \ldots, n-1$ satisfying the following commutation relations:

$$
\begin{aligned}
& {\left[p_{\mu}, p_{\nu}\right]=0,} \\
& {\left[p_{\mu}, \hat{x}^{\nu}\right]=-i \varphi_{\mu}{ }^{\nu}\left(\frac{p}{M}\right),} \\
& {\left[\hat{x}^{\mu}, \hat{x}^{\nu}\right]=\frac{i}{M} \hat{x}^{\alpha} C^{\mu \nu}{ }_{\alpha}\left(\frac{p}{M}\right),}
\end{aligned}
$$

where $M$ is the mass parameter, $\varphi_{\mu \nu}\left(\frac{p}{M}\right)$ is an invertible matrix, and $C_{\mu \nu}{ }^{\alpha}\left(\frac{p}{M}\right)=-C_{\nu \mu}{ }^{\alpha}\left(\frac{p}{M}\right)$ are generalized structure constants depending on the momenta [36].

The matrix $\varphi_{\mu}{ }^{\nu}$ is arbitrary and the structure constants $C^{\mu \nu}{ }_{\alpha}$ are restricted by the Jacobi relations which include the matrix $\varphi_{\mu}{ }^{\nu}$. In the limit $M \rightarrow \infty$, the matrix $\varphi_{\mu \nu} \rightarrow \eta_{\mu \nu}$ and $\left[\hat{x}_{\mu}, \hat{x}_{\nu}\right] \rightarrow 0$, where $\eta_{\mu \nu}=\operatorname{diag}(-1,1, \ldots, 1)$, or more generally, instead of $\eta_{\mu \nu}$, we have the metric $g_{\mu \nu}$ with an arbitrary signature.

The deformed phase spaces, Eq. (1), generalize Lie algebras. Note that if the $\hat{x}^{\mu}$ generate a given Lie algebra with structure constants $C^{\mu \nu}{ }_{\lambda}$, then there are infinitely many possible matrices $\varphi_{\mu}{ }^{\nu}$, compatible with the Jacobi relations [36]; see also [37-39].
In the following, we shall put/fix $M=1$. Now we consider the most general matrix $\varphi_{\mu}{ }^{v}$ describing vector-like deformations defined by vector $u^{\mu}, u^{2} \in\{-1,0,1\}$, i.e. time-, lightand space-like, respectively [40];

$\varphi_{\mu}^{\nu}(p)=\delta_{\mu}^{v} f_{1}+u_{\mu} p^{v} f_{2}+u_{\mu} u^{v} f_{3}+u^{v} p_{\mu} f_{4}+p_{\mu} p^{v} f_{5}$

where $f_{1, \ldots, 5}$ are functions of $A=u \cdot p$ and $B=p^{2}$.

In order to fullfil the commutation relations $\left[p_{\mu}, \hat{x}^{\nu}\right]=$ $-i \varphi_{\mu}{ }^{\nu}(p)$, Eq. (1), we consider a realization of $\hat{x}^{\mu}$ of the form

$$
\begin{aligned}
\hat{x}^{\mu}= & x^{\alpha} \varphi_{\alpha}{ }^{\mu}=x^{\mu} f_{1}+(u \cdot x) p^{\mu} f_{2}+(u \cdot x) u^{\mu} f_{3} \\
& +(x \cdot p) u^{\mu} f_{4}+(x \cdot p) p^{\mu} f_{5}
\end{aligned}
$$

where $x^{\mu}$ are commutative coordinates conjugate to $p_{\mu}$, i.e.

$$
\begin{aligned}
& {\left[x^{\mu}, x^{\nu}\right]=0,} \\
& {\left[p_{\mu}, x^{\nu}\right]=-i \delta_{\mu}^{\nu},} \\
& {\left[p_{\mu}, p_{\nu}\right]=0,}
\end{aligned}
$$

describing the undeformed phase space Heisenberg algebra $\mathcal{H}$. The undeformed coordinates $x_{\mu}$ generate an enveloping algebra $\mathcal{A}$, which is a subalgebra of the undeformed Heisenberg algebra, i.e. $\mathcal{A} \subset \mathcal{H}$. The momenta $p_{\mu}$ generate the algebra $\mathcal{T}$, which is also a subalgebra of the undeformed Heisenberg algebra, i.e. $\mathcal{T} \subset \mathcal{H}$. The undeformed Heisenberg algebra is, symbolically, $\mathcal{H}=\mathcal{A} \mathcal{T}$.

Then the structure of the commutation relations $\left[\hat{x}^{\mu}, \hat{x}^{\nu}\right]$ is given by

$$
\begin{aligned}
{\left[\hat{x}^{\mu}, \hat{x}^{\nu}\right]=} & i\left[\left(u^{\mu} \hat{x}^{\nu}-u^{\nu} \hat{x}^{\mu}\right) F_{1}+\left(\hat{x}^{\mu} p^{\nu}-\hat{x}^{v} p^{\mu}\right) F_{2}\right. \\
& +(u \cdot \hat{x})\left(u^{\mu} p^{\nu}-u^{\nu} p^{\mu}\right) F_{3} \\
& \left.+(\hat{x} \cdot p)\left(u^{\mu} p^{\nu}-u^{\nu} p^{\mu}\right) F_{4}\right]
\end{aligned}
$$

where $F_{1, \ldots, 4}$ are also functions of $A$ and $B$ which can be expressed in terms of the functions $f_{1, \ldots, 5}$ and their derivatives.

We point out that this construction, Eqs. (2) and (5), unifies commutative spaces with $\varphi_{\mu}{ }^{\nu} \neq \delta_{\mu}^{\nu}$, as well as various types of NC spaces, including $\kappa$-Minkowski space [13,14], Snyder type spaces [41-43] and $\kappa$-Snyder spaces [44,45]. Moyal type spaces ( $\theta$-deformation) [4] could also be included in this construction by adding $\chi^{\mu}(p)$ in the realization of $\hat{x}^{\mu}$, i.e. $\hat{x}^{\mu}=x^{\alpha} \varphi_{\alpha}^{\mu}(p)+\chi^{\mu}(p)$. For example, the simplest realization of Moyal space is $\hat{x}^{\mu}=x^{\mu}-\frac{1}{2} \theta^{\mu \alpha} p_{\alpha}$, where $\theta^{\mu \nu} \in \mathbb{R}$ is an antisymmetric tensor.

Note that quadratic algebras cannot be included in the above construction and a new generalization is required. 


\section{Star product and twist operator}

The action $\triangleright$ is defined by

$x^{\mu} \triangleright f(x)=x^{\mu} f(x)$,

$p_{\mu} \triangleright f(x)=-i \frac{\partial f}{\partial x^{\mu}}$, i.e. $p_{\mu}=-i \frac{\partial}{\partial x^{\mu}} \equiv-i \partial_{\mu}$.

Then it follows that [35]

$$
\begin{aligned}
\hat{x}^{\mu} \triangleright 1 & =x^{\mu}, \\
e^{i k \cdot \hat{x}} \triangleright e^{i q \cdot x} & =e^{i \mathcal{P}(k, q) \cdot x}, \quad k_{\mu}, q_{\mu} \in \mathcal{M}_{1, n-1},
\end{aligned}
$$

where $\mathcal{M}_{1, n-1}$ is Minkowski momentum space and $\mathcal{P}_{\mu}(k, q)$ satisfies the differential equation

$\frac{\mathrm{d} \mathcal{P}_{\mu}(\lambda k, q)}{\mathrm{d} \lambda}=k_{\alpha} \varphi_{\mu}{ }^{\alpha}(\mathcal{P}(\lambda k, q))$

with $\mathcal{P}_{\mu}(k, 0)=K_{\mu}(k), \mathcal{P}_{\mu}(0, q)=q_{\mu}$ and $\lambda \in \mathbb{R}$.

Hence,

$e^{i k \cdot \hat{x}} \triangleright 1=e^{i K(k) \cdot x}$

and

$e^{i K^{-1}(k) \cdot \hat{x}} \triangleright 1=e^{i k \cdot x}$,

where $K^{-1}(k)$ is the inverse map of $K_{\mu}(k)$, i.e. $K_{\mu}^{-1}$ $(K(k))=k_{\mu}$.

The star product is defined by [35]

$e^{i k \cdot x} \star e^{i q \cdot x}=e^{i K^{-1}(k) \cdot \hat{x}} \triangleright e^{i q \cdot x}=e^{i \mathcal{D}(k, q) \cdot x}$,

where

$\mathcal{D}_{\mu}(k, q)=\mathcal{P}_{\mu}\left(K^{-1}(k), q\right)$,

$\mathcal{D}_{\mu}(k, 0)=k_{\mu}, \quad \mathcal{D}_{\mu}(0, q)=q_{\mu}$.

The deformed addition of momenta is defined by

$(k \oplus q)_{\mu}=\mathcal{D}_{\mu}(k, q)$.

The coproduct $\Delta p_{\mu}$ is

$\Delta p_{\mu}=\mathcal{D}_{\mu}(p \otimes 1,1 \otimes p)$

For functions $f(x)$ and $g(x)$ which can be Fourier transformed, the relation between star product and twist operator is given by $[46,47]$

$(f \star g)(x)=m\left[\mathcal{F}^{-1}(\triangleright \otimes \triangleright)(f(x) \otimes g(x))\right]$, where $m$ is a map $m: \mathcal{H} \otimes \mathcal{H} \rightarrow \mathcal{H}$ such that $m\left(h_{1} \otimes h_{2}\right)=$ $h_{1} h_{2}$ with $h_{1}, h_{2} \in \mathcal{H}$ and

$\mathcal{F}^{-1}=: e^{i\left(1 \otimes x^{\alpha}\right)\left(\Delta-\Delta_{0}\right) p_{\alpha}}:+\mathcal{I}_{0}$

where $\Delta_{0} p_{\mu}=p_{\mu} \otimes 1+1 \otimes p_{\mu}$ and $\mathcal{I}_{0}$ is the right ideal defined by ${ }^{1}$

$m\left[\mathcal{I}_{0}(\triangleright \otimes \triangleright)(f(x) \otimes g(x))\right]=0$.

The symbol : $:$ denotes the normal ordering in which the $x$ stand to the left of the $p$.

If the generators $\hat{x}^{\mu}$ close the subalgebra, i.e. if the commutator $\left[\hat{x}^{\mu}, \hat{x}^{\nu}\right]$ does not depend on the momenta, then the PBW theorem holds, the star product is associative and the coproduct is coassociative. The inverse statement also holds. If the star product is associative, the twist operator Eq. (18) satisfies the cocycle condition in the Hopf algebroid sense and vice versa [47-52].

We also have

$$
\begin{aligned}
& \Delta p_{\mu}=\mathcal{F} \Delta_{0} p_{\mu} \mathcal{F}^{-1}=\mathcal{D}_{\mu}(p \otimes 1,1 \otimes p), \\
& \hat{x}^{\mu}=m\left[\mathcal{F}^{-1}(\triangleright \otimes 1)\left(x^{\mu} \otimes 1\right)\right] \\
& \quad=x^{\mu}+i x^{\alpha} m\left[\left(\Delta-\Delta_{0}\right) p_{\alpha}(\triangleright \otimes 1)\left(x^{\mu} \otimes 1\right)\right] \\
& \quad=x^{\alpha} \varphi_{\alpha}^{\mu}(p), \\
& e^{i K^{-1}(k) \cdot \hat{x}}=m\left[\mathcal{F}^{-1}(\triangleright \otimes 1)\left(e^{i k \cdot x} \otimes 1\right)\right] .
\end{aligned}
$$

The above construction generalizes Sect. 4 in [35] to nonassociative star products. Note that the commutator $\left[p_{\mu}, \hat{x}^{\nu}\right]$ is given by

$\left[p_{\mu}, \hat{x}^{\nu}\right]=-i \delta_{\mu}^{\nu}+m\left\{\left[\Delta_{0} p_{\mu}, \mathcal{F}^{-1}\right](\triangleright \otimes 1)\left(x^{\nu} \otimes 1\right)\right\}$.

\section{Linear realizations and twists}

In this section, we consider linear realizations of vector-like deformed phase space, that is, the realizations where the function $\varphi_{\mu}{ }^{v}(p)$ is linear in the momenta,

$$
\begin{aligned}
\varphi_{\mu}{ }^{v}(p)= & \delta_{\mu}^{v}+c_{1} \delta_{\mu}^{v}(u \cdot p)+c_{2} u^{v} p_{\mu}+c_{3} u_{\mu} u^{v}(u \cdot p) \\
& +c_{4} u_{\mu} p^{v}=\delta_{\mu}^{v}+K_{\mu}^{\alpha \nu} p_{\alpha}
\end{aligned}
$$

where $K_{\mu}^{\alpha \nu} \in \mathbb{R}$ is proportional to the deformation scale $1 / M$. In terms of $c_{1, \ldots, 4}, \hat{x}^{\mu}=x^{\alpha} \varphi_{\alpha}^{\mu}(p)$ and $K_{\mu}^{\alpha v}$ are given by

$$
\begin{aligned}
& \hat{x}^{\mu}=x^{\mu}\left(1+c_{1}(u \cdot p)\right)+c_{2} u^{\mu}(x \cdot p) \\
& \quad+c_{3} u^{\mu}(u \cdot x)(u \cdot p)+c_{4}(u \cdot x) p^{\mu}, \\
& K^{\alpha \nu}{ }_{\mu}=c_{1} \delta_{\mu}^{v} u^{\alpha}+c_{2} u^{v} \delta_{\mu}^{\alpha}+c_{3} u^{\alpha} u^{v} u_{\mu}+c_{4} \eta^{\alpha v} u_{\mu} .
\end{aligned}
$$

\footnotetext{
${ }^{1}$ The ideal $\mathcal{I}_{0}(19)$ is generated by $x_{\mu} \otimes 1-1 \otimes x_{\mu}$
} 
Since $\hat{x}^{\mu}=x^{\alpha} \varphi_{\alpha}{ }^{\mu}(p)=x^{\mu}+K^{\beta \mu}{ }_{\alpha} x^{\alpha} p_{\beta}$, then it follows that

$$
\begin{aligned}
{\left[\hat{x}^{\mu}, \hat{x}^{\nu}\right]=} & \left(K^{\mu \nu}{ }_{\alpha}-K^{v \mu}{ }_{\alpha}\right) \hat{x}^{\alpha} \\
& +i\left[K^{\beta \mu}{ }_{\alpha} K^{\alpha \nu}{ }_{\gamma}-K^{\beta \nu}{ }_{\alpha} K^{\alpha \mu}{ }_{\gamma}\right. \\
& \left.-\left(K^{\mu \nu}{ }_{\alpha}-K^{\nu \mu}{ }_{\alpha}\right) K^{\beta \alpha}{ }_{\gamma}\right] L^{\gamma}{ }_{\beta},
\end{aligned}
$$

where $L^{\mu}{ }_{\nu}=x^{\mu} p_{\nu}$. The generators $L^{\mu}{ }_{v}$ and the momenta $p_{\mu}$ generate the $\mathfrak{i g l}(n)$ algebra:

$$
\begin{aligned}
& {\left[L^{\alpha}{ }_{\beta}, L_{\delta}^{\gamma}\right]=i\left(\delta_{\delta}^{\alpha} L_{\beta}^{\gamma}-\delta_{\beta}^{\gamma} L_{\delta}^{\alpha}\right)} \\
& {\left[L_{\nu}^{\mu}, p_{\lambda}\right]=i \delta_{\lambda}^{\mu} p_{\nu}}
\end{aligned}
$$

and

$\left[L^{\mu}{ }_{\nu}, x^{\lambda}\right]=-i \delta_{v}^{\lambda} x^{\mu}$.

The generators $\hat{x}^{\mu}$ and $L^{\mu}{ }_{\nu}$ close a Lie algebra,

$\left[L^{\mu}{ }_{\nu}, \hat{x}^{\lambda}\right]=-i \delta_{v}^{\lambda} \hat{x}^{\mu}+i\left(\delta_{v}^{\lambda} K^{\beta \mu}{ }_{\alpha}+\delta_{v}^{\beta} K_{\alpha}^{\mu \lambda}-\delta_{\alpha}^{\mu} K^{\beta \lambda}{ }_{\nu}\right) L^{\alpha}{ }_{\beta}$.

The Lie algebra is closed in $\hat{x}^{\mu}$ if

$K_{\lambda}^{\beta \mu} K_{\alpha}^{\lambda v}-K_{\lambda}^{\beta \nu} K_{\alpha}^{\lambda \mu}=\left(K_{\lambda}^{\mu \nu}-K_{\lambda}^{v \mu}\right) K_{\alpha}^{\beta \lambda}$

and $C^{\mu \nu}{ }_{\lambda}=K_{\lambda}^{\mu \nu}{ }_{\lambda}-K^{v \mu}{ }_{\lambda}$ are structure constants, i.e. $\left[\hat{x}^{\mu}, \hat{x}^{\nu}\right]=i C^{\mu \nu} \lambda_{\lambda} \hat{x}^{\lambda}$.

Application of Eqs. (10) and (14) to the algebra $\left\{\hat{x}^{\mu}, L^{\mu}{ }_{\nu}\right\}$ gives

$\mathcal{P}_{\mu}(k, q)=\left(\frac{1-e^{-\mathcal{K}(k)}}{\mathcal{K}(k)}\right){ }^{\alpha}{ }_{\mu} k_{\alpha}+\left(e^{-\mathcal{K}(k)}\right)^{\alpha}{ }_{\mu} q_{\alpha}$,

where $\mathcal{K}(k)^{\mu}{ }_{\nu}=-K^{\mu \alpha}{ }_{\nu} k_{\alpha}$. Specially,

$K_{\mu}(k)=\mathcal{P}_{\mu}(k, 0)=\left(\frac{1-e^{-\mathcal{K}(k)}}{\mathcal{K}(k)}\right){ }^{\alpha}{ }_{\mu} k_{\alpha}$.

The function $\mathcal{D}_{\mu}(k, q)$ is given by

$\mathcal{D}_{\mu}(k, q)=\mathcal{P}_{\mu}\left(K^{-1}(k), q\right)=k_{\mu}+\left(e^{-\mathcal{K}\left(K^{-1}(k)\right)}\right){ }^{\alpha}{ }_{\mu} q_{\alpha}$,

which defines the deformed addition of momenta. For linear realizations, the star product is associative if and only if condition (31) is satisfied. Up to the second order in the deformation, the function $\mathcal{D}_{\mu}(k, q)$ is given by

$$
\begin{aligned}
\mathcal{D}_{\mu}(k, q)= & k_{\mu}+q_{\mu}+K^{\beta \alpha}{ }_{\mu} k_{\alpha} q_{\beta} \\
& +\frac{1}{2}\left(K_{\lambda}^{\gamma \beta} K^{\lambda \alpha}{ }_{\mu}-K_{\lambda}^{\alpha \beta} K_{\mu}^{\gamma \lambda}\right) k_{\alpha} k_{\beta} q_{\gamma} \\
& +\mathcal{O}\left(1 / M^{3}\right) .
\end{aligned}
$$

It is straightforward to show that the deformed addition of momenta $(k \oplus q)_{\mu}=\mathcal{D}_{\mu}(k, q)$ is associative in the first order and in order to be associative in the second order, condition (31) has to be satisfied, which also implies a Lie algebra closed in $\hat{x}^{\mu}$. The Lie algebra closed in $\hat{x}^{\mu}$ leads to the associative star product, which leads to an associative $\mathcal{D}_{\mu}(k, q)$, which implies that for linear realizations $(k \oplus q)_{\mu}=\mathcal{D}_{\mu}(k, q)$ is associative in all orders if and only if the condition (31) is satisfied.

The deformed coproduct of the momenta $\Delta: \mathcal{T} \rightarrow \mathcal{T} \otimes \mathcal{T}$ is

$$
\Delta p_{\mu}=\mathcal{D}_{\mu}(p \otimes 1,1 \otimes p)=p_{\mu} \otimes 1+\left(e^{-\mathcal{K}\left(p^{W}\right)}\right)^{\alpha}{ }_{\mu} \otimes p_{\alpha},
$$

where $p_{\mu}^{W}=K_{\mu}^{-1}(p)$ and it is a function of the momenta with the property

$\left(p_{\mu}^{W}-k_{\mu}\right) e^{i k \cdot \hat{x}} \triangleright 1=0$,

where $\mathrm{W}$ stands for Weyl ordering. For details of the calculation of $p_{\mu}^{W}$, see Appendix A. It follows that $\Delta p_{\mu}$ is coassociative for linear realizations of the non-commutative coordinates $\hat{x}^{\mu}$ if and only if $\hat{x}^{\mu}$ close a Lie algebra, i.e. if condition (31) holds.

Note that the commutator $\left[L^{\mu}{ }_{\nu}, \hat{x}^{\lambda}\right]$ is given by

$\left[L^{\mu}{ }_{\nu}, \hat{x}^{\lambda}\right]=-i \delta_{\nu}^{\lambda} \hat{x}^{\mu}+m\left\{\left[\Delta_{0} L^{\mu}{ }_{\nu}, \mathcal{F}^{-1}\right](\triangleright \otimes 1)\left(x^{\lambda} \otimes 1\right)\right\}$.

\subsection{Twist and R-matrix}

Combining Eq. (18) for the twist and Eq. (36) for the deformed coproduct of momenta yields

$\mathcal{F}^{-1}=: \exp \left\{i\left(e^{\mathcal{K}\left(p^{W}\right)}-1\right)^{\beta}{ }_{\alpha} \otimes x^{\alpha} p_{\beta}\right\}:$.

Furthermore, it can be shown that

$$
: e^{i A^{\beta}{ }_{\alpha} x^{\alpha}} p_{\beta}:=e^{i[\ln (1+A)]_{\alpha}^{\beta} x^{\alpha} p_{\beta}}
$$

holds for any $A_{\alpha}^{\beta}$ such that $\left[A^{\alpha}{ }_{\beta}, A^{\gamma}{ }_{\delta}\right]=0$ and $\left[A^{\alpha}{ }_{\beta}, x^{\gamma}\right.$ $\left.p_{\delta}\right]=0$; see Appendix B from [35]. Using the identity (40), we find

$\mathcal{F}^{-1}=\exp \left(-i \mathcal{K}\left(p^{W}\right)^{\beta}{ }_{\alpha} \otimes x^{\alpha} p_{\beta}\right)=\exp \left(i p_{\alpha}^{W} \otimes\left(\hat{x}^{\alpha}-x^{\alpha}\right)\right)$.

Twist (39) is written in Hopf algebroid approach [46,47]. The main point is that it can be written in the standard form (41), where $x_{\alpha} p_{\beta}$ is identified with the $\mathfrak{g l}(n)$ generators $L_{\alpha \beta}$, satisfying (28). 
The twist (41) satisfies the normalization condition

$m(\epsilon \otimes 1) \mathcal{F}=1=m(1 \otimes \epsilon) \mathcal{F}$.

For linear realizations $\hat{x}_{\mu}$ that close a Lie algebra $\left[\hat{x}^{\mu}, \hat{x}^{\nu}\right]=i\left(c_{1}-c_{2}\right)\left(u^{\mu} \hat{x}^{\nu}-u^{\nu} \hat{x}^{\mu}\right)$, the twist (41) will satisfy the cocycle condition for arbitrary choice of vector $u_{\mu}$

$(\mathcal{F} \otimes 1)\left(\Delta_{0} \otimes 1\right) \mathcal{F}=(1 \otimes \mathcal{F})\left(1 \otimes \Delta_{0}\right) \mathcal{F}$

The proof is analogous to the one provided in [35]. Generally, if condition (31) is not satisfied, twist (41) will not satisfy the cocycle condition (43).

The R-matrix is given by [46] (see also [53])

$\mathcal{R}=\tilde{\mathcal{F}} \mathcal{F}^{-1}=e^{-\left(\hat{x}^{\alpha}-x^{\alpha}\right) \otimes i p_{\alpha}^{W}} e^{i p_{\beta}^{W} \otimes\left(\hat{x}^{\beta}-x^{\beta}\right)}$,

where $\tilde{\mathcal{F}}=\exp \left(-\left(\hat{x}^{\alpha}-x^{\alpha}\right) \otimes i p_{\alpha}^{W}\right)$ is the transposed twist $\tilde{\mathcal{F}}=\tau_{0} \mathcal{F} \tau_{0}$, where $\tau_{0}: \mathcal{H} \otimes \mathcal{H} \rightarrow \mathcal{H} \otimes \mathcal{H}$ is a linear map such that $\tau_{0}(A \otimes B)=B \otimes A \forall A, B \in \mathcal{H}$.

In the case of commutative spaces with $\left[p_{\mu}, \hat{x}^{\nu}\right] \neq-i \delta_{\mu}^{\nu}$, the coproducts $\Delta p_{\mu}$ are cocommutative, the star product is commutative, $\mathcal{R}-1 \otimes 1 \in \mathcal{I}_{0}$ and $\mathcal{F}^{-1}-\tilde{\mathcal{F}}^{-1} \in \mathcal{I}_{0}$ (see Eq. (19) for the definition of the right ideal $\mathcal{I}_{0}$ ).

The R-matrix is given by $\mathcal{R}=1 \otimes 1+r_{c l}+\mathcal{O}\left(1 / M^{2}\right)$, where $r_{c l}=i K^{\beta \alpha}{ }_{\gamma}\left(p_{\alpha} \otimes L^{\gamma}{ }_{\beta}-L_{\beta}^{\gamma} \otimes p_{\alpha}\right)$ is the classical r-matrix, which will satisfy the Yang-Baxter equation if and only if the condition (31) is satisfied.

The twisted flip operator $\tau$ is defined by

$\tau=\mathcal{F}_{\tau_{0}} \mathcal{F}^{-1}=\tau_{0} \mathcal{R}$

and it satisfies the following properties:

$[\Delta h, \tau]=0, \quad \forall h \in \mathcal{H}$,

$\tau^{2}=1 \otimes 1$.

Projector operators for the twisted symmetric and antisymmetric sectors of the Hilbert space are given by $\frac{1}{2}(1 \otimes 1 \pm \tau)$. Using twisted flip operator, the bosonic state is defined by

$f \otimes g=\tau(f \otimes g)$

or equivalently

$\mathcal{F}^{-1}(\triangleright \otimes \triangleright)(f \otimes g)=\tilde{\mathcal{F}}^{-1}(\triangleright \otimes \triangleright)(g \otimes f)$.

Note that the bosonic state remains invariant under the action of the projector operator $\frac{1}{2}(1 \otimes 1+\tau)$ for the twisted symmetric sector of the Hilbert space.

\section{Three types of star products from linear realizations}

In this section, we present star products which are: (i) commutative and associative (Sect. 5.1), (ii) non-commutative and associative (Sect. 5.2) and (iii) non-commutative and non-associative (Sect. 5.3).

\subsection{Commutative spaces with $\left[p_{\mu}, \hat{x}^{v}\right] \neq-i \delta_{\mu}^{v}$}

Using linear realizations for $\hat{x}_{\mu}=x^{\alpha} \varphi_{\alpha \mu}(p)$ with $\varphi_{\mu \nu}(p)$ given in Eq. (24), and using Eq. (27) restricted to the commutative case, i.e. $\left[\hat{x}_{\mu}, \hat{x}_{v}\right]=0$, we find three families of solutions for commutative spaces. We have

(i) : $\hat{x}^{\mu}=x^{\mu}+c_{3} u^{\mu}(u \cdot x)(u \cdot p), \quad c_{1}=c_{2}=c_{4}=0$,

$$
\begin{aligned}
\text { (ii) : } \hat{x}^{\mu}= & x^{\mu}+c_{3}\left[u^{\mu}(u \cdot x)(u \cdot p)-u^{2}(u \cdot x) p^{\mu}\right], \\
c_{1} & =c_{2}=0, c_{4}=-c_{3} \\
\text { (iii) }: \quad \hat{x}_{\mu}= & x^{\mu}\left[1-c_{3} u^{2}(u \cdot p)\right] \\
& +c_{3} u^{\mu}\left[(u \cdot x)(u \cdot p)-u^{2}(x \cdot p)\right] \\
c_{1} & =c_{2}=-c_{3}, c_{4}=0
\end{aligned}
$$

For the sake of simplicity, $c \equiv c_{3}$ will be used in the rest of this subsection.

For the family (i),

$$
\begin{aligned}
& K_{\mu}(k)=\left\{\begin{array}{l}
k_{\mu}+\left(\frac{e^{c(u \cdot k) u^{2}}-1}{c(u \cdot k) u^{2}}-1\right) \frac{u \cdot k}{u^{2}} u_{\mu}, \quad u^{2} \neq 0 \\
k_{\mu}+\frac{1}{2} c(u \cdot k)^{2} u_{\mu}, \quad u^{2}=0
\end{array}\right. \\
& K_{\mu}^{-1}(k)= \begin{cases}k_{\mu}-c \frac{c u^{2}(u \cdot k)-\ln \left[1+c u^{2}(u \cdot k)\right]}{\left(c u^{2}\right)^{2}} u_{\mu}, \quad u^{2} \neq 0 \\
k_{\mu}-c \frac{(u \cdot k)^{2}}{2} u_{\mu}, & u^{2}=0 .\end{cases}
\end{aligned}
$$

Functions $\mathcal{P}_{\mu}(k, q)$ and $\mathcal{D}_{\mu}(k, q)$ for the family $\left.i\right)$ are given by

$\mathcal{P}_{\mu}(k, q)=\left\{\begin{array}{c}k_{\mu}+q_{\mu}+c(u \cdot k)\left[\left(\frac{e^{c u^{2}(u \cdot k)}-1}{c u^{2}(u \cdot k)}-1\right) \frac{1}{c u^{2}}\right. \\ \left.\quad+\frac{e^{c u^{2}(u \cdot k)}-1}{c u^{2}(u \cdot k)}(u \cdot q)\right] u_{\mu}, \quad u^{2} \neq 0, \\ k_{\mu}+q_{\mu}+c(u \cdot k)\left(\frac{u \cdot k}{2}+u \cdot q\right) u_{\mu}, \quad u^{2}=0,\end{array}\right.$

$\mathcal{D}_{\mu}(k, q)=k_{\mu}+q_{\mu}+c(u \cdot k)(u \cdot q) u_{\mu}$. 
For the family (ii),

$$
\begin{aligned}
& K_{\mu}(k)=k_{\mu}+\frac{c}{2}\left[(u \cdot k)^{2}-u^{2} k^{2}\right] u_{\mu}, \\
& K_{\mu}^{-1}(k)=k_{\mu}-\frac{c}{2}\left[(u \cdot k)^{2}-u^{2} k^{2}\right] u_{\mu} .
\end{aligned}
$$

Furthermore,

$$
\begin{aligned}
\mathcal{P}_{\mu}(k, q)= & k_{\mu}+q_{\mu}+c u_{\mu}\left[\frac{(u \cdot k)^{2}-u^{2} k^{2}}{2}\right. \\
& \left.+(u \cdot k)(u \cdot q)-u^{2}(k \cdot q)\right], \\
\mathcal{D}_{\mu}(k, q)= & k_{\mu}+q_{\mu}+c u_{\mu}\left[(u \cdot k)(u \cdot q)-u^{2}(k \cdot q)\right] .
\end{aligned}
$$

For the family (iii),

$$
\begin{aligned}
& K_{\mu}(k)=\left\{\begin{array}{l}
e^{-c u^{2}(u \cdot k)} k_{\mu}+\frac{1-e^{-c u^{2}(u \cdot k)}\left(1+c u^{2}(u \cdot k)\right)}{c\left(u^{2}\right)^{2}} u_{\mu}, \quad u^{2} \neq 0, \\
k_{\mu}+\frac{1}{2} c(u \cdot k)^{2} u_{\mu}, \quad u^{2}=0,
\end{array}\right. \\
& K_{\mu}^{-1}(k)= \begin{cases}\frac{k_{\mu}}{1-c u^{2}(u \cdot k)} & \begin{array}{l}
-c\left(u u^{2}(u \cdot k)-\left(1-c u^{2}(u \cdot k)\right) \ln \left(1-c u^{2}(u \cdot k)\right)\right. \\
+c(u \cdot k)^{2}
\end{array} u_{\mu}, \quad u^{2} \neq 0, \\
k_{\mu}-c \frac{(u \cdot k)^{2}}{2} u_{\mu}, & u^{2}=0,\end{cases}
\end{aligned}
$$

$$
\begin{aligned}
P_{\mu}(k, q)= & K_{\mu}(k)+q_{\mu}-c u^{2}\left[K_{\mu}(k)(u \cdot q)+q_{\mu}(u \cdot K(k))\right] \\
& +c u_{\mu}(u \cdot K(k))(u \cdot q), \\
\mathcal{D}_{\mu}(k, q)= & k_{\mu}+q_{\mu}-c u^{2}\left[k_{\mu}(u \cdot q)\right. \\
& \left.+q_{\mu}(u \cdot k)\right]+c u_{\mu}(u \cdot k)(u \cdot q) .
\end{aligned}
$$

Note that for each family of commutative spaces, $\mathcal{D}_{\mu}(k, q)=$ $\mathcal{D}_{\mu}(q, k)$, which implies commutativity of the corresponding star products and cocommutativity of the corresponding coproducts. Also, for each of these families, $\mathcal{D}_{\mu}\left(\mathcal{D}\left(k_{1}, k_{2}\right)\right.$, $\left.k_{3}\right)=\mathcal{D}_{\mu}\left(k_{1}, \mathcal{D}\left(k_{2}, k_{3}\right)\right)$, which implies associativity of the corresponding star products and coassociativity of the corresponding coproducts, which is consistent with $\left[\hat{x}^{\mu}, \hat{x}^{\nu}\right]=0$.

\section{$5.2 \kappa$-Minkowski spaces}

There are four families of linear realizations of $\kappa$-Minkowski space. Their classification is given in [35]:

$$
\begin{aligned}
\mathcal{C}_{1}: \quad \hat{x}^{\mu}= & x^{\mu}+\left[c_{2}(x \cdot p)+c(u \cdot x)(u \cdot p)\right] u^{\mu}, \\
\mathcal{C}_{2}: \quad \hat{x}^{\mu}= & x^{\mu}+c_{1} x^{\mu}(u \cdot p) \\
& +c[(u \cdot x)(u \cdot p)-(x \cdot p)] u^{\mu}, \\
\mathcal{C}_{3}: \quad \hat{x}^{\mu}= & x^{\mu}+\left[c_{2}(x \cdot p)+c(u \cdot x)(u \cdot p)\right] u^{\mu} \\
& +\left(c_{2}-c\right)(u \cdot x) p^{\mu}, \\
\mathcal{C}_{4}: \quad \hat{x}^{\mu}= & x^{\mu}+c_{1}\left[x^{\mu}(u \cdot p)-(u \cdot x) p^{\mu}\right], \\
& \quad \text { only for } u^{2}=0 .
\end{aligned}
$$

The family $\mathcal{C}_{4}$ was also considered in $[54,55]$. For each family, the parameters $c_{1, \ldots, 4}$ are given by

$\mathcal{C}_{1}: \quad c_{1}=0, \quad c_{2} \in \mathbb{R}, \quad c_{3}=c, \quad c_{4}=0$,

$\mathcal{C}_{2}: \quad c_{1} \in \mathbb{R} \quad c_{2}=-c, \quad c_{3}=c, \quad c_{4}=0$,

$\mathcal{C}_{3}: \quad c_{1}=0, \quad c_{2} \in \mathbb{R}, \quad c_{3}=c, \quad c_{4}=c_{2}-c$,

$\mathcal{C}_{4}: \quad c_{1} \in \mathbb{R}, \quad c_{2}=0, \quad c_{3}=0, \quad c_{4}=-c_{1}$.

We point out that $c=0$ for $u^{2}=0$ and $c \in \mathbb{R}$ for $u^{2} \neq 0$. The commutator of the coordinates is given by

$\left[\hat{x}^{\mu}, \hat{x}^{\nu}\right]=i\left(c_{1}-c_{2}\right)\left(u^{\mu} \hat{x}^{\nu}-u^{\nu} \hat{x}^{\mu}\right) \equiv i\left(a^{\mu} \hat{x}^{\nu}-a^{\nu} \hat{x}^{\mu}\right)$,

where $a^{\mu}=\left(c_{1}-c_{2}\right) u^{\mu}$.

Explicitly, for $\mathcal{C}_{1}, \mathcal{C}_{2}, \mathcal{C}_{3}$ and $\mathcal{C}_{4}$, the functions $K_{\mu}^{-1}(k)$ and $\mathcal{D}_{\mu}(k, q)$ are given by

- Case $\mathcal{C}_{1}$ :

$$
K_{\mu}^{-1}(k)=\left\{\begin{array}{l}
{\left[k_{\mu}-\frac{a_{\mu}}{a^{2}}(Z(k)-1-a \cdot k)\right] \frac{\ln Z(k)}{Z(k)-1}, \quad a^{2} \neq 0,} \\
k_{\mu} \frac{\ln Z(k)}{Z(k)-1}, \quad a^{2}=0,
\end{array}\right.
$$

$$
\mathcal{D}_{\mu}(k, q)=\left\{\begin{array}{l}
k_{\mu}+Z(k) q_{\mu}+\frac{a_{\mu}}{a^{2}}\left(Z(k)^{1-c}-Z(k)\right)(a \cdot q), \quad a^{2} \neq 0, \\
k_{\mu}+Z(k) q_{\mu}, \quad a^{2}=0,
\end{array}\right.
$$

where

$$
Z(k)=[1-(1-c) a \cdot k]^{\frac{1}{1-c}} .
$$

\section{- Case $\mathcal{C}_{2}$ :}

$$
\begin{aligned}
& K_{\mu}^{-1}(k)=\left\{\begin{array}{l}
{\left[k_{\mu}-\frac{a_{\mu}}{a^{2}}\left(1-Z(k)^{-1}+a \cdot k\right)\right]} \\
\quad \times \frac{\ln Z(k)}{1-Z(k)^{-1}}, \quad a^{2} \neq 0, \\
k_{\mu} \frac{\ln Z(k)}{1-Z(k)^{-1}}, \quad a^{2}=0,
\end{array}\right. \\
& \mathcal{D}_{\mu}(k, q)=\left\{\begin{array}{l}
k_{\mu}+\left(Z(k)^{c}-\frac{c}{1+c}\right) q_{\mu}+\left(c \frac{a_{\mu}}{a^{2}}+(c-1) \frac{K_{\mu}^{-1}(k)}{\ln Z(k)}\right) \\
\times \frac{Z(k)^{-1}-Z(k)^{c}}{1+c} a \cdot q, \quad a^{2} \neq 0, \\
k_{\mu}+q_{\mu}-K^{-1}(k) \frac{Z(k)^{-1}-1}{\ln Z(k)} a \cdot q, \quad a^{2}=0,
\end{array}\right.
\end{aligned}
$$

where

$$
Z(k)=[1-(c-1) a \cdot k]^{\frac{c}{c-1}} .
$$


- Case $\mathcal{C}_{3}$ :

$$
\begin{aligned}
& K_{\mu}^{-1}(k)=\left\{\begin{array}{l}
{\left[k_{\mu}-\frac{a_{\mu}}{a^{2}}(Z(k)-1+a \cdot k)\right]} \\
\times \frac{\ln Z(k)}{Z(k)-1}, \quad a^{2} \neq 0, \\
{\left[k_{\mu}+\frac{a_{\mu} k^{2}}{Z(k)}\right] \frac{\ln Z(k)}{Z(k)-1}, \quad a^{2}=0,}
\end{array}\right. \\
& \mathcal{D}_{\mu}(k, q)=\left\{\begin{array}{c}
k_{\mu}+Z(k) q_{\mu}+a_{\mu}\left((1+c) \frac{K^{-1}(k) \cdot q}{\ln Z(k)}-c \frac{a \cdot q}{a^{2}}\right) \\
\times(Z(k)-1) Z(k), \quad a^{2} \neq 0, \\
k_{\mu}+Z(k) q_{\mu} \\
+a_{\mu} \frac{K^{-1}(k) \cdot q}{\ln Z(k)}(Z(k)-1) Z(k), \quad a^{2}=0,
\end{array}\right.
\end{aligned}
$$

where

$$
Z(k)=\left[c+(1-c)\left((1-a \cdot k)^{2}-a^{2} k^{2}\right)\right]^{\frac{1}{2(1-c)}} .
$$

\section{- Case $\mathcal{C}_{4}$ :}

$$
\begin{gathered}
K_{\mu}^{-1}(k)=\left(k_{\mu}+\frac{a_{\mu}}{2} k^{2}\right) \frac{\ln Z(k)}{1-Z(k)^{-1}}, \\
\mathcal{D}_{\mu}(k, q)=k_{\mu} Z(q)^{-1}+q_{\mu}-a_{\mu}(k \cdot q) Z(k) \\
-\frac{a_{\mu}}{2} k^{2} Z(k)(a \cdot k),
\end{gathered}
$$

where

$$
Z(k)=\frac{1}{1+a \cdot k} .
$$

Note that for each of these families, $\mathcal{D}_{\mu}\left(\mathcal{D}\left(k_{1}, k_{2}\right), k_{3}\right)=$ $\mathcal{D}_{\mu}\left(k_{1}, \mathcal{D}\left(k_{2}, k_{3}\right)\right)$, which implies associativity of the corresponding star products and coassociativity of the corresponding coproducts, which is consistent with $\hat{x}^{\mu}$ closing a Lie algebra, i.e. $\left[\hat{x}^{\mu}, \hat{x}^{\nu}\right]=i C^{\mu \nu}{ }_{\lambda} \hat{x}^{\lambda}$. In this case, the structure constants $C_{\lambda}^{\mu \nu}$ are given by $C_{\lambda}^{\mu \nu}=a^{\mu} \delta_{\lambda}^{\nu}-a^{\nu} \delta_{\lambda}^{\mu}$.

We note that the Jordanian twist, leading to $\kappa$-Minkowski space [56], produces linear realizations $\hat{x}^{\mu}=x^{\mu}(1+a \cdot p)$, which belongs to the case $\mathcal{C}_{2}$. A new construction of a simple interpolation between two Jordanian twists, corresponding to linear realizations $\mathcal{C}_{1}$ and $\mathcal{C}_{2}$, was proposed in [57].

\subsection{Deformed phase spaces generated by Poincaré-Weyl generators $/ \kappa$-Snyder spaces}

Here, we consider the algebras with $c_{3}=0$ and $c_{4}=-c_{1}$, i.e. the realization is

$$
\begin{aligned}
\hat{x}_{\mu} & =x_{\mu}\left(1+c_{1}(u \cdot p)\right)-c_{1}(u \cdot x) p_{\mu}+c_{2} u_{\mu}(x \cdot p) \\
& =x_{\mu}-c_{1} u^{\alpha} M_{\alpha \mu}+c_{2} u_{\mu} D
\end{aligned}
$$

where $M_{\mu \nu}=L_{\mu \nu}-L_{\nu \mu}$ are the Lorentz generators and $D=x \cdot p$ is the dilatation operator.

The algebra of generators $\hat{x}_{\mu}, M_{\mu \nu}$ and $D$ is given by

$$
\begin{aligned}
& {\left[\hat{x}_{\mu}, \hat{x}_{\nu}\right]=i\left(c_{1}-c_{2}\right)\left(u_{\mu} \hat{x}_{\nu}-u_{\nu} \hat{x}_{\mu}\right)} \\
& \quad+i c_{1}\left[c_{1} u^{2} M_{\mu \nu}-c_{2} u^{\alpha}\left(u_{\mu} M_{\alpha \nu}-u_{\nu} M_{\alpha \mu}\right)\right] \\
& \quad\left[M_{\mu \nu}, \hat{x}_{\lambda}\right]=i\left[\eta_{\mu \lambda} \hat{x}_{\nu}-\eta_{\nu \lambda} \hat{x}_{\mu}\right. \\
& \left.\quad+c_{1}\left(M_{\mu \lambda} u_{\nu}-M_{\nu \lambda} u_{\mu}\right)-c_{2}\left(\eta_{\mu \lambda} u_{\nu}-\eta_{\nu \lambda} u_{\mu}\right) D\right] \\
& {\left[D, \hat{x}_{\mu}\right]=-i \hat{x}_{\mu}+i c_{1} u^{\alpha} M_{\alpha \mu}-i c_{2} u_{\mu} D} \\
& {\left[M_{\mu \nu}, M_{\rho \tau}\right]=i\left(\eta_{\mu \rho} M_{\nu \tau}-\eta_{\nu \rho} M_{\mu \tau}-\eta_{\mu \tau} M_{\nu \rho}+\eta_{\nu \tau} M_{\mu \rho}\right)} \\
& {\left[M_{\mu \nu}, D\right]=0 .}
\end{aligned}
$$

For the realization (86), the functions $K_{\mu}(k), K_{\mu}^{-1}(k)$, $\mathcal{P}_{\mu}(k, q)$ and $\mathcal{D}_{\mu}(k, q)$ are

$$
\begin{aligned}
& K_{\mu}(k)=k_{\mu}+\frac{1}{2}\left[\left(c_{1}+c_{2}\right)(u \cdot k) k_{\mu}-c_{1} k^{2} u_{\mu}\right] \\
& \quad+\frac{1}{6}\left[\left(c_{1}+c_{2}\right)^{2}(u \cdot k)^{2}-c_{1}^{2} k^{2} u^{2}\right] k_{\mu} \\
& \quad-\frac{1}{3} c_{1} c_{2}(u \cdot k) k^{2} u_{\mu}+\mathcal{O}\left(1 / M^{3}\right), \\
& K_{\mu}^{-1}(k)=k_{\mu}-\frac{1}{2}\left[\left(c_{1}+c_{2}\right)(u \cdot k) k_{\mu}-c_{1} k^{2} u_{\mu}\right] \\
& \quad+\left[\frac{\left(c_{1}+c_{2}\right)^{2}}{3}(u \cdot k)^{2}-\frac{c_{1}}{4}\left(\frac{c_{1}}{3}+c_{2}\right) k^{2} u^{2}\right] k_{\mu} \\
& \quad-\frac{c_{1}}{4}\left(c_{1}+\frac{5 c_{2}}{3}\right)(u \cdot k) k^{2} u_{\mu}+\mathcal{O}\left(1 / M^{3}\right), \\
& \mathcal{P}_{\mu}(k, q)=K_{\mu}(k)+q_{\mu}+c_{1}\left[k_{\mu}(u \cdot q)-(k \cdot q) u_{\mu}\right] \\
& \quad+c_{2}(u \cdot k) q_{\mu}+\mathcal{O}\left(1 / M^{2}\right), \\
& \mathcal{D}_{\mu}(k, q)=k_{\mu}+q_{\mu}+c_{1}\left[k_{\mu}(u \cdot q)-(k \cdot q) u_{\mu}\right] \\
& \quad+c_{2}(u \cdot k) q_{\mu}+\mathcal{O}\left(1 / M^{2}\right) .
\end{aligned}
$$

The function $\mathcal{D}_{\mu}(k, q)$ is associative only in two cases-in the case $c_{1}=0$, which corresponds to realization (65) ( $\kappa$ Minkowski $\mathcal{C}_{1}$ ) with $c=0$, and in the case $c_{2}=0, u^{2}=0$, which corresponds to the realization (68) $\left(\kappa\right.$-Minkowski $\left.\mathcal{C}_{4}\right)$.

There are special cases of deformed phase space generated by Poincaré generators appearing only in $1+1,2+1$ and $3+1$ spacetime dimensions. Their realizations are given by

$1+1: \quad \hat{x}^{\mu}=x^{\mu}+c u^{\mu} \epsilon^{\alpha \beta} M_{\alpha \beta}$,

$2+1: \quad \hat{x}^{\mu}=x^{\mu}+c u^{\mu} \epsilon^{\alpha \beta \gamma} M_{\alpha \beta} u_{\gamma}$,

$3+1: \quad \hat{x}^{\mu}=x^{\mu}+c \epsilon^{\mu \alpha \beta \gamma} M_{\alpha \beta} u_{\gamma}$,

where $\epsilon^{\alpha \beta}, \epsilon^{\alpha \beta \gamma}$ and $\epsilon^{\alpha \beta \gamma \delta}$ are the Levi-Civita tensors for $1+1,2+1$ and $3+1$ dimensions, respectively. Commutators of coordinates $\hat{x}^{\mu}$ are given by 
$1+1: \quad\left[\hat{x}^{\mu}, \hat{x}^{\nu}\right]=2 i c\left[\left(\epsilon^{\alpha \mu} u^{\nu}-\epsilon^{\alpha \nu} u^{\mu}\right) \hat{x}_{\alpha}+2 c u^{2} M^{\mu \nu}\right]$,

$2+1: \quad\left[\hat{x}^{\mu}, \hat{x}^{\nu}\right]=2 i c u_{\alpha}\left(\epsilon^{\alpha \beta \mu} u^{\nu}-\epsilon^{\alpha \beta v} u^{\mu}\right) \hat{x}_{\beta}$,

$3+1: \quad\left[\hat{x}^{\mu}, \hat{x}^{\nu}\right]=4 i c \epsilon^{\mu \nu \alpha \beta} \hat{x}_{\alpha} u_{\beta}+4 i c^{2} u^{2} M^{\mu \nu}$.

$\kappa$-Snyder spaces defined by $\hat{x}^{\mu}=x^{\mu}+c u_{\alpha} M^{\alpha \mu}, u^{2} \neq 0$ were considered in $[44,45]$.

\section{Twisted symmetry algebras}

In an undeformed $\mathfrak{i g l}(n)$ Hopf algebra, coproducts $\Delta_{0}$ : $\mathfrak{i g l}(n) \rightarrow \mathfrak{i g l}(n) \otimes \mathfrak{i g l}(n)$, counit $\epsilon: \mathfrak{i g l}(n) \rightarrow \mathbb{C}$ and antipode $S_{0}: \mathfrak{i g l}(n) \rightarrow \mathfrak{i g l} l(n)$ are given by

$\Delta_{0} p_{\mu}=p_{\mu} \otimes 1+1 \otimes p_{\mu}$,

$\Delta_{0} L_{\mu \nu}=L_{\mu \nu} \otimes 1+1 \otimes L_{\mu \nu}$,

$\epsilon\left(p_{\mu}\right)=\epsilon\left(L_{\mu \nu}\right)=0$,

$\epsilon(1)=1, S_{0}\left(p_{\mu}\right)=-p_{\mu}, \quad S_{0}\left(L_{\mu \nu}\right)=-L_{\mu \nu}$.

When applied to undeformed $\operatorname{igl}(n)$ algebra, the twist (41) produces the corresponding deformed $\mathfrak{i g l}(n)$ Hopf algebras or generalized Hopf algebras (quasi-bialgebras). For $h \in \mathfrak{i g l}(n)$, the deformed coproduct $\Delta h$ is given by

$\Delta h=\mathcal{F} \Delta_{0} h \mathcal{F}^{-1}$

where $\Delta_{0} h$ is the undeformed coproduct of $h$.

The antipode $S(h)$ is obtained from the coproduct $\Delta h$ using the identity

$m[(S \otimes 1) \Delta h]=m[(1 \otimes S) \Delta h]=\epsilon(h)$,

where $\epsilon(h)$ is the counit, which remains undeformed.

Coproducts and antipodes of $p_{\mu}$ and $L^{\mu}{ }_{\nu}$ are given by

$$
\begin{aligned}
& \Delta p_{\mu}=\mathcal{F} \Delta_{0} p_{\mu} \mathcal{F}^{-1}=p_{\mu} \otimes 1+\left(e^{\mathcal{K}}\right)^{\alpha}{ }_{\mu} \otimes p_{\alpha} \\
& \Delta L^{\mu}{ }_{\nu}=\mathcal{F} \Delta_{0} L^{\mu}{ }_{\nu} \mathcal{F}^{-1}=L^{\mu}{ }_{\nu} \otimes 1+\left(\left(e^{-\mathcal{K}}\right)^{\beta} \gamma \frac{\partial\left(e^{\mathcal{K}}\right)^{\gamma} \alpha}{\partial p_{\mu}} p_{\nu}\right. \\
& \quad+\left(e^{-\mathcal{K})^{\beta}}{ }_{\nu}\left(e^{\mathcal{K}}\right)^{\mu}{ }_{\alpha}\right) \otimes L^{\alpha}{ }_{\beta} \\
& S\left(p_{\mu}\right)=-\left(e^{-\mathcal{K}}\right)^{\alpha}{ }_{\mu} p_{\alpha} \\
& S\left(L^{\mu}{ }_{\nu}\right)=-\left(\left(e^{\mathcal{K}}\right)^{\beta} \gamma \frac{\partial\left(e^{-\mathcal{K}}\right)^{\gamma}{ }_{\alpha}}{\partial S\left(p_{\mu}\right)} S\left(p_{\nu}\right)\right. \\
& \left.\quad+\left(e^{\mathcal{K}}\right)^{\beta}{ }_{\nu}\left(e^{-\mathcal{K}}\right)^{\mu}{ }_{\alpha}\right) L^{\alpha}{ }_{\beta} .
\end{aligned}
$$

For the family $i$ ) of commutative spaces (Sect. 5.1), the coproduct and the antipode of $p_{\mu}$ are given by

$\Delta p_{\mu}=\Delta_{0} p_{\mu}+c u_{\mu}(u \cdot p) \otimes(u \cdot p)$,

$S\left(p_{\mu}\right)=-p_{\mu}-c u_{\mu} \frac{(u \cdot p)^{2}}{1+c u^{2}(u \cdot p)}$.

For the family (ii) of commutative spaces (Sect. 5.1), the coproduct and the antipode of $p_{\mu}$ are given by

$\Delta p_{\mu}=\Delta_{0} p_{\mu}+c u_{\mu}\left[(u \cdot p) \otimes(u \cdot p)-u^{2} p_{\alpha} \otimes p^{\alpha}\right]$,

$S\left(p_{\mu}\right)=-p_{\mu}-c u_{\mu}\left[(u \cdot p)^{2}-u^{2} p^{2}\right]$.

For the family (iii) of commutative spaces (Sect. 5.1), the coproduct and the antipode of $p_{\mu}$ are given by

$$
\begin{aligned}
\Delta p_{\mu}= & \Delta_{0} p_{\mu}+c u_{\mu}(u \cdot p) \otimes(u \cdot p)-c u^{2}\left[(u \cdot p) \otimes p_{\mu}\right. \\
& \left.+p_{\mu} \otimes(u \cdot p)\right], \\
S\left(p_{\mu}\right) & =-\frac{p_{\mu}}{1-c u^{2}(u \cdot p)}+c u_{\mu}\left(\frac{u \cdot p}{1-c u^{2}(u \cdot p)}\right)^{2} .
\end{aligned}
$$

For the families $\mathcal{C}_{1,2,3,4}$ of $\kappa$-Minkowski spaces (65), (66), (67) and (68), presented in Sect. 5.2, coproducts and antipodes of $p_{\mu}$ and $L^{\mu}{ }_{\nu}$ are presented in [35].

For deformed phase spaces generated by the PoincaréWeyl generators (86), presented in Sect. 5.3, the coproducts are coassociative only in two cases-case $c_{1}=0$ and case $c_{2}=0$ with $u^{2}=0$-which correspond to $\kappa$ Minkowski space. Otherwise, these deformed phase spaces lead to a generalized Hopf algebra (quasi-bialgebra) with non-coassociative coproducts. For these deformed phase spaces, the coproduct and antipode of $p_{\mu}$ are given by

$$
\begin{aligned}
\Delta p_{\mu}= & \Delta_{0} p_{\mu}+c_{1}\left(p_{\mu} \otimes u \cdot p-u_{\mu} p_{\alpha} \otimes p^{\alpha}\right) \\
& +c_{2}(u \cdot p) \otimes p_{\mu}+\mathcal{O}\left(1 / M^{2}\right), \\
S\left(p_{\mu}\right)= & -p_{\mu}\left(1-\left(c_{1}+c_{2}\right)(u \cdot p)\right)-c_{1} u_{\mu} p^{2}+\mathcal{O}\left(1 / M^{2}\right) .
\end{aligned}
$$

\section{Transposed twists and left-right dual algebras}

The transposed twist $\tilde{\mathcal{F}}=\tau_{0} \mathcal{F} \tau_{0}$, obtained from $\mathcal{F}$ by interchanging left and right side of the tensor product, is given by

$\tilde{\mathcal{F}}=\exp \left(\left(\hat{x}^{\alpha}-x^{\alpha}\right) \otimes\left(-i p_{\alpha}^{W}\right)\right)$.

Twist $\tilde{\mathcal{F}}$ will be a Drinfeld twist, satisfying cocycle and normalization condition, if and only if this also holds for the 
twist $\mathcal{F}$. From the transposed twist $\tilde{\mathcal{F}}$, a set of dual noncommutative coordinates can be obtained,

$\hat{y}^{\mu}=m\left[\tilde{\mathcal{F}}^{-1}(\triangleright \otimes 1)\left(x^{\mu} \otimes 1\right)\right]=x^{\alpha}\left(e^{-\mathcal{K}\left(p^{W}\right)}\right)^{\mu}{ }_{\alpha}$.

In the second order of deformation, dual coordinates $\hat{y}^{\mu}$ are given by

$$
\begin{aligned}
\hat{y}^{\mu}= & x^{\mu}+K_{\alpha}^{\mu \beta} x^{\alpha} p_{\beta} \\
& +\frac{1}{2}\left(K_{\lambda}^{\mu \beta} K_{\alpha}^{\lambda \gamma}-K_{\alpha}^{\mu \lambda} K_{\lambda}^{\beta \gamma}\right) x^{\alpha} p_{\beta} p_{\gamma} \\
& +\mathcal{O}\left(1 / M^{3}\right) .
\end{aligned}
$$

Commutators $\left[\hat{x}^{\mu}, \hat{y}^{\nu}\right]$ and $\left[\hat{y}^{\mu}, \hat{y}^{\nu}\right]$ are given by

$$
\begin{aligned}
& {\left[\hat{x}^{\mu}, \hat{y}^{\nu}\right]=\frac{i}{2}\left[\left(K_{\lambda}^{\beta \mu}-K_{\lambda}^{\mu \beta}\right) K_{\alpha \lambda}^{\nu \lambda}\right.} \\
& \left.+K_{\lambda}^{\nu \mu} K_{\alpha}^{\lambda \beta}-K_{\lambda}^{\nu \beta} K_{\alpha}^{\lambda \mu}\right] x^{\alpha} p_{\beta}+\mathcal{O}\left(1 / M^{3}\right) \\
& \equiv \frac{i}{2} T_{\alpha \mu \nu}^{\beta x^{\beta}} p_{\alpha}+\mathcal{O}\left(1 / M^{3}\right) \text {, } \\
& {\left[\hat{y}^{\mu}, \hat{y}^{\nu}\right]=-i\left(K_{\lambda}^{\mu \nu}-K_{\lambda}^{\nu \mu}\right) \hat{y}^{\lambda}+\frac{i}{2}\left[\left(K_{\lambda}^{\mu \nu}-K_{\lambda}^{\nu \mu}\right) K_{\alpha}^{\lambda \beta}\right.} \\
& +\left(K_{\lambda}^{\mu \beta}-K_{\lambda}^{\beta \mu}\right)\left(K_{\alpha}^{\nu \lambda}-K_{\alpha}^{\lambda \nu}\right)-\left(K_{\lambda}^{\mu \beta}\right. \\
& \left.-K_{\lambda}^{\beta \mu}\right)\left(K_{\alpha}^{\nu \lambda}-K_{\alpha}^{\lambda \nu}\right) \\
& \left.-K_{\lambda}^{\beta \mu} K_{\alpha}^{\lambda \nu}+K_{\lambda}^{\beta \nu} K_{\alpha}^{\lambda \mu}\right] x^{\alpha} p_{\beta}+\mathcal{O}\left(1 / M^{3}\right) \\
& =-i\left(K_{\lambda}^{\mu \nu}-K_{\lambda}^{\nu \mu}\right) \hat{y}^{\lambda} \\
& +\frac{i}{2}\left(T_{\alpha \beta}^{\mu \beta}-T_{\alpha \beta \mu_{\alpha}}^{\nu x^{\alpha}} p_{\beta}+\mathcal{O}\left(1 / M^{3}\right),\right.
\end{aligned}
$$

where $T^{\mu \nu \beta_{\alpha}}$ is given by

$$
T_{\alpha}^{\mu \nu \beta}=\left(K_{\lambda}^{\mu \nu}-K_{\lambda}^{\nu \mu}\right) K_{\alpha}^{\beta \lambda}+K_{\lambda}^{\beta \nu} K_{\alpha}^{\lambda \mu}-K_{\lambda}^{\beta \mu} K_{\alpha}^{\lambda \nu} .
$$

The condition $T^{\mu \nu \beta}{ }_{\alpha}=0$ is equivalent to the condition (31), which corresponds to the case of a Lie-algebraic deformation.

For commutative spaces (50)-(52), coproducts are cocommutative, therefore $\tilde{\mathcal{F}}$ and $\mathcal{F}$ are equivalent, i.e. $\mathcal{F}^{-1}-\tilde{\mathcal{F}}^{-1} \in$ $\mathcal{I}_{0}$, and the result is trivial: $\hat{y}^{\mu}=\hat{x}^{\mu}$. For the special cases of $\kappa$-Minkowski spaces, the results for $\hat{y}^{\mu}$ are given in Sect. VII of [35]. For Lie-algebraic deformations, the noncommutative coordinates $\hat{x}^{\mu}$ commute with their duals $\hat{y}^{\mu}$,

$\left[\hat{x}^{\mu}, \hat{y}^{\nu}\right]=0$

and their duals also close a Lie algebra,

$$
\left[\hat{y}^{\mu}, \hat{y}^{v}\right]=-i C_{\lambda v}^{\mu \nu} \hat{y}^{\lambda}
$$

For deformed phase spaces generated by the PoincaréWeyl generators (86), the result for $\hat{y}^{\mu}$ to second order is

$$
\begin{aligned}
\hat{y}^{\mu}= & x^{\mu}+c_{1}\left(u^{\mu}(x \cdot p)-(u \cdot x) p^{\mu}\right)+c_{2} x^{\mu}(u \cdot p) \\
& +\frac{c_{1}}{2}\left[c_{1}\left(2(u \cdot x)(u \cdot p) p^{\mu}-u^{\mu}(u \cdot x) p^{2}-u^{2}(x \cdot p) p^{\mu}\right)\right. \\
& \left.+c_{2}\left(x^{\mu}(u \cdot p)^{2}-x^{\mu} u^{2} p^{2}+u^{\mu}(x \cdot p)(u \cdot p)-(u \cdot x)(u \cdot p) p^{\mu}\right)\right] \\
& +\mathcal{O}\left(1 / M^{3}\right) .
\end{aligned}
$$

The generators (86) fail to commute with their duals in the second order,

$$
\begin{aligned}
{\left[\hat{x}^{\mu}, \hat{y}^{\nu}\right]=} & i \frac{c_{1}}{2}\left\{c_{1} u^{2}\left((x \cdot p) \eta^{\mu \nu}-x^{\mu} p^{v}\right)\right. \\
& +c_{2}\left[\left(x^{\mu}(u \cdot p)-u^{\mu}(x \cdot p)\right) u^{v}\right. \\
& \left.\left.-(u \cdot x)\left((u \cdot p) \eta^{\mu \nu}-u^{\mu} p^{v}\right)\right]\right\} \\
& +\mathcal{O}\left(1 / M^{3}\right)
\end{aligned}
$$

and the commutator of their duals is given by

$$
\begin{aligned}
{\left[\hat{y}^{\mu}, \hat{y}^{v}\right]=} & i\left(c_{2}-c_{1}\right)\left(u^{\mu} \hat{y}^{v}-u^{v} \hat{y}^{\mu}\right) \\
& +i \frac{c_{1}}{2}\left\{c_{1} u^{2}\left(x^{\mu} p^{v}-x^{v} p^{\mu}\right)\right. \\
& +c_{2}\left[\left(u^{\mu} x^{v}-u^{v} x^{\mu}\right)(u \cdot p)\right. \\
& \left.\left.-(u \cdot x)\left(u^{\mu} \eta^{\beta v}-u^{v} \eta^{\beta \mu}\right)\right]+\mathcal{O}\left(1 / M^{3}\right)\right\} \\
= & i\left(c_{2}-c_{1}\right)\left(u^{\mu} \hat{y}^{v}-u^{v} \hat{y}^{\mu}\right) \\
& +c_{1}\left[c_{1} u^{2} M^{\mu v}+c_{2} u_{\alpha}\left(u^{\mu} M^{\alpha v}-u^{v} M^{\alpha \mu}\right)\right] \\
& +\mathcal{O}\left(1 / M^{3}\right) .
\end{aligned}
$$

\section{Outlook and discussion}

Families of vector-like deformed relativistic quantum phase spaces and corresponding realizations are analyzed. A method for a general construction of the star product is presented. The corresponding twist, expressed in terms of the phase space coordinates, in the Hopf algebroid sense is presented. General linear realizations are considered and corresponding twists, in terms of the momenta and the PoincaréWeyl generators or $\mathfrak{g l}(n)$ generators, are constructed and the R-matrix is discussed. A classification of linear realizations leading to vector-like deformed phase spaces is given. There are three types of spaces: (i) commutative spaces, (ii) $\kappa$-Minkowski spaces and (iii) $\kappa$-Snyder spaces. The corresponding star products are (i) associative and commutative (but non-local), (ii) associative and non-commutative and (iii) non-associative and non-commutative, respectively. Twisted symmetry algebras are considered. The transposed twists and left-right dual algebras are presented.

In this paper, we were dealing mostly with linear realizations and corresponding twists. In commutative spaces (Sect. 5.1) and $\kappa$-Minkowski spaces (Sect. 5.2), i.e. in Lie-deformed Minkowski spaces, linear realizations lead to Drinfeld twists 
satisfying the cocycle and normalization conditions. In $\kappa$ Snyder spaces (Sect. 5.3), the star product is non-associative and the twist does not satisfy the cocycle condition. Field theories defined on spaces with non-associative star products are constructed; see for example versions on $\kappa$-Snyder space $[44,45]$ and on Snyder space [42,58-60]. The properties of field theories on non-associative star products are currently under investigation. In [40], a phenomenological analysis related to vector-like deformations of the relativistic quantum phase space and relativistic kinematics was elaborated up to first order in the deformation, particularly on particle propagation in spacetime. Note that if NC coordinates $\hat{x}_{\mu}$ close a Lie algebra in $\hat{x}_{\mu}$, then the corresponding deformed quantum phase space has a Hopf algebroid structure [4649]. Otherwise, the coproduct is non-coassociative and the corresponding structure should be quasi-bialgebroid. A generalization of the Hopf algebroid which includes an antipode is under investigation. The corresponding symmetry algebra is a certain deformation of the $\mathfrak{i g l}(n)$ Hopf algebra. This new framework is more suitable for addressing questions of quantum gravity [62] and related new effects of Planck scale physics.

We point out that in all Lie-deformed Minkowski spaces, the problem of finding all possible linear realizations is closely related to the classification of bicovariant differential calculi on $\kappa$-Minkowski space [55]. Namely, the requirement that differential calculus is bicovariant leads to finding all possible Lie superalgebras generated by non-commutative coordinates and non-commutative one-forms. The corresponding equations for the structure constants from super Jacobi identities are the same as (31). Linear realizations expressed in terms of the Heisenberg algebra can be extended to a super Heisenberg algebra by introducing Grassman coordinates and momenta. The corresponding extended twists generate whole differential calculi. In [63], a new class of linear realizations leading to Lie-deformed Minkowski spaces has been proposed and the related twisted statistics properties have been considered.

It is much easier to understand and to perform practical calculations in the non-commutative space with a linear realization of non-commutative coordinates. In [64-66] it is proposed that the non-commutative metric should be a central element of the whole differential algebra and that it should encode some of the main properties of the quantum theory of gravity. Linear realizations might provide a way to perform such calculations for a large class of deformations, and for all types of bicovariant differential calculi and predict new contributions to the physics of quantum black holes and the quantum origin of the cosmological constant $[67,68]$.

Acknowledgements The work by S.M. and D.P. has been supported by the Croatian Science Foundation under the Project No. IP-2014-09-
9582 as well as by the H2020 Twinning Project No. 692194, "RBI-TWINNING".

Open Access This article is distributed under the terms of the Creative Commons Attribution 4.0 International License (http://creativecomm ons.org/licenses/by/4.0/), which permits unrestricted use, distribution, and reproduction in any medium, provided you give appropriate credit to the original author(s) and the source, provide a link to the Creative Commons license, and indicate if changes were made.

Funded by $\mathrm{SCOAP}^{3}$.

\section{A Calculation of $p_{\mu}^{W}$}

The momentum $p_{\mu}^{W}$ is calculated from

$p_{\mu}=\left(\frac{1-e^{-\mathcal{K}}}{\mathcal{K}}\right){ }^{\alpha}{ }_{\mu} p_{\alpha}^{W}$.

Multiplying by the inverse matrix leads to

$p_{\mu}^{W}=\left(\frac{\mathcal{K}}{1-e^{-\mathcal{K}}}\right)^{\alpha}{ }_{\mu} p_{\alpha}$

or, order by order,

$p_{\mu}^{W}=\left(1+\frac{\mathcal{K}\left(p^{W}\right)}{2}+\frac{\mathcal{K}\left(p^{W}\right)^{2}}{12}-\frac{\mathcal{K}\left(p^{W}\right)^{4}}{720}+\mathcal{O}\left(\mathcal{K}^{6}\right)\right){ }^{\alpha}{ }_{\mu} p_{\alpha}$.

The first few terms in the expansion are

$\left(p_{(0)}^{W}\right)_{\mu}=p_{\mu}$,

$\left(p_{(1)}^{W}\right)_{\mu}=\frac{\mathcal{K}^{\alpha}{ }_{\mu}(p)}{2} p_{\alpha}$,

$\left(p_{(2)}^{W}\right)_{\mu}=\left(\frac{\mathcal{K}(\mathcal{K}(p) p)}{4}+\frac{\mathcal{K}(p)^{2}}{12}\right){ }^{\alpha}{ }_{\mu} p_{\alpha}$,

$\left(p_{(3)}^{W}\right)_{\mu}=\left(\frac{\mathcal{K}(\mathcal{K}(\mathcal{K}(p) p) p)}{8}+\frac{\mathcal{K}\left(\mathcal{K}(p)^{2} p\right)}{24}\right.$

$\left.+\frac{\mathcal{K}(p) \mathcal{K}(\mathcal{K}(p) p)}{24}+\frac{\mathcal{K}(\mathcal{K}(p) p) \mathcal{K}(p)}{24}\right)^{\alpha}{ }_{\mu} p_{\alpha}$.

For $K_{\mu \nu \alpha}$ leading to non-commutative coordinates $\hat{x}^{\mu}$ that close a Lie algebra, i.e. $\left[\hat{x}^{\mu}, \hat{x}^{\nu}\right]=i C^{\mu \nu}{ }_{\lambda} \hat{x}^{\lambda}$, this can be written without nesting:

$$
\begin{aligned}
\left(p_{(0)}^{W}\right)_{\mu} & =p_{\mu}, \\
\left(p_{(1)}^{W}\right)_{\mu} & =\frac{1}{2} \mathcal{K}^{\alpha}{ }_{\mu} p_{\alpha}, \\
\left(p_{(2)}^{W}\right)_{\mu} & =\left[\left(\frac{\mathcal{K}}{3}-\frac{\mathcal{C}}{4}\right) \mathcal{K}\right]{ }_{\mu}^{\alpha} p_{\alpha}, \\
\left(p_{(3)}^{W}\right)_{\mu} & =\left[\left(\frac{\mathcal{K}}{2}-\frac{\mathcal{C}}{3}\right)\left(\mathcal{K}-\frac{\mathcal{C}}{2}\right) \frac{\mathcal{K}}{2}\right]{ }_{\mu}{ }_{\mu} p_{\alpha},
\end{aligned}
$$


where $\mathcal{K}^{\mu}{ }_{v}=-K^{\mu \alpha}{ }_{\nu} p_{\alpha}$ and $\mathcal{C}^{\mu}{ }_{\nu}=-C^{\mu \alpha}{ }_{\nu} p_{\alpha}$, where $C^{\mu \alpha}{ }_{v}=K^{\mu \alpha}{ }_{v}-K^{\alpha \mu}{ }_{v}$ are structure constants. For example, for linear realizations of commutative coordinates, i.e. $\left[\hat{x}^{\mu}, \hat{x}^{\nu}\right]=0, p_{\mu}^{W}$ is given by

$p_{\mu}^{W}=\left[\frac{-\ln (1-\mathcal{K})}{\mathcal{K}}\right]{ }^{\alpha}{ }_{\mu} p_{\alpha}$.

For $\kappa$-Minkowski space, see [35].

\section{References}

1. S. Doplicher, K. Fredenhagen, J.E. Roberts, Spacetime quantization induced by classical gravity. Phys. Lett. B 331, 39 (1994)

2. S. Doplicher, K. Fredenhagen, J.E. Roberts, The quantum structure of spacetime at the Planck scale and quantum fields. Commun. Math. Phys. 172, 187 (1995)

3. S. Majid, Foundations of Quantum Group Theory (Cambridge University Press, Cambridge, 1995)

4. J .E. Moyal, Quantum Mechanics as a Statistical Theory Mathematical Proceedings of the Cambridge Philosophical Society (Cambridge University Press, Cambridge, 1949), pp. 99-124

5. J. Lukierski, H. Ruegg, Quantum kappa Poincaré in any dimension. Phys. Lett. B 329, 189 (1994). arXiv:hep-th/9310117

6. J. Lukierski, A. Nowicki, H. Ruegg, V.N. Tolstoy, Q-deformation of Poincaré algebra. Phys. Lett. B 264, 331 (1991)

7. J. Lukierski, A. Nowicki, H. Ruegg, New quantum Poincaré algebra and k-deformed field theory. Phys. Lett. B 293, 344 (1992)

8. J. Kowalski-Glikman, S. Nowak, Doubly special relativity theories as different bases of $\kappa$-Poincaré algebra. Phys. Lett. B 539, 126 (2002). arXiv:hep-th/0203040

9. G. Amelino-Camelia, Doubly-special relativity: first results and key open problems. Int. J. Mod. Phys. D 11, 35 (2002). arXiv:gr-qc/0210063

10. G. Amelino-Camelia, Testable scenario for Relativity with minimum-length. Phys. Lett. B 510, 255 (2001). arXiv:hep-th/0012238

11. D. Kovačević, S. Meljanac, A. Pachoł, R. Štrajn, Generalized Poincare algebras, Hopf algebras and kappa-Minkowski spacetime. Phys. Lett. B 711, 122-127 (2012). arXiv:1202.3305 [hep-th]

12. A. Connes, Noncommutative Geometry (Academic Press, New York, 1994)

13. S. Majid, H. Ruegg, Bicrossproduct structure of $\kappa$-Poincaré group and non-commutative geometry. Phys. Lett. B 334, 348-354 (1994). arXiv:hep-th/9405107

14. J. Lukierski, H. Ruegg, W.J. Zakrzewski, Classical and quantum mechanics of free $\kappa$-relativistic systems. Ann. Phys. 243, 90-116 (1995). arXiv:hep-th/9312153

15. P. Kosinski, P. Maslanka, J. Lukierski, A. Sitarz, Generalized kappa-deformations and deformed relativistic scalar fields on noncommutative Minkowski Space. Proceedings of the Conference Topics in Mathematical Physics, General Relativity and Cosmology, World Scientific, Singapore (2003). arXiv:hep-th/0307038

16. J. Lukierski, V. Lyakhovsky, M. Mozrzymas, $\kappa$-deformations of $D=4$ Weyl and conformal symmetries. Phys. Lett. B 538, 375 (2002). arXiv:hep-th/0203182

17. P. Kosiński, J. Lukierski, P. Maślanka, $\kappa$-deformed Wigner construction of relativistic wave functions and free fields on $\kappa$ Minkowski space. Nucl. Phys. Proc. Suppl. 102, 161-168 (2001). arXiv:hep-th/0103127
18. J. Lukierski, in Proceedings of Alushta Conference on Recent Problems in QFT, May 1996, ed. D. Shirkov, D. I. Kazakov A. A. Vladimirov, Dubna, p. 82 (1996)

19. S. Pramanik, S. Ghosh, P. Pal, Electrodynamics of a generalized charged particle in doubly special relativity framework. Ann. Phys. 346, 113 (2014). arXiv:1212.6881 [hep-th]

20. S. Ghosh, P. Pal, Deformed special relativity and deformed symmetries in a canonical framework. Phys. Rev. D 75, 105021 (2007). arXiv:hep-th/0702159

21. T.R. Govindarajan, K.S. Gupta, E. Harikumar, S. Meljanac, D. Meljanac, Deformed oscillator algebras and QFT in $\kappa$-Minkowski spacetime. Phys. Rev. D 80, 025014 (2009). arXiv:0903.2355 [hepth]

22. S. Meljanac, A. Samsarov, J. Trampetić, M. Wohlgenannt, Scalar field propagation in the $\phi^{4}$ kappa-Minkowski model. JHEP 1112 (2011). arXiv:1111.5553 [hep-th]

23. F.A. Bais, N.M. Muller, B.J. Schroers, Quantum group symmetry and particle scattering in $(2+1)$-dimensional quantum gravity. Nucl. Phys. B 640, 3-45 (2002). arXiv:hep-th/0205021

24. C. Meusburger, B. Schroers, Generalised Chern-Simons actions for $3 d$ gravity and $\kappa$-Poincaré symmetry. Nucl. Phys. B 806, 462-488 (2009). arXiv:0805.3318 [hep-th]

25. G. Amelino-Camelia, L. Smolin, A. Starodubtsev, Quantum symmetry, the cosmological constant and Planck scale phenomenology Class. Quant. Gravit. 21, 3095 (2004). arXiv:hep-th/0306134

26. L. Freidel, J. Kowalski-Glikman, L. Smolin, $2+1$ gravity and Doubly special relativity. Phys. Rev. D 69, 044001 (2004). arXiv:hep-th/0307085

27. L. Freidel, E.R. Livine, 3d Quantum gravity and effective noncommutative quantum field theory. Phys. Rev. Lett. 96, 221301 (2006). arXiv:hep-th/0512113

28. M. Chaichian, A. Demichev, Introduction to Quantum Groups (World Scientific, Singapore, 1996)

29. J. Madore, An Introduction to Noncommutative Differential Geometry and its Physical Applications (London Mathematical Society Lecture Note Series) (Cambridge University Press, Cambridge, 1999)

30. V.G. Drinfeld, Quantum groups. Proceedings of the ICM, Rhode Island (1987)

31. P. Aschieri, M. Dimitrijević, P. Kulish, F. Lizzi, J. Wess, Noncommutative Spacetimes: Symmetries in Noncommutative Geometry and Field Theory (Lecture Notes in Physics) (Springer, New York, 2009)

32. P. Aschieri, F. Lizzi, P. Vitale, Twisting all the way: from classical mechanics to quantum fields. Phys. Rev. D 77, 025037 (2008). arXiv:0708.3002 [hep-th]

33. V.G. Drinfeld, Hopf algebras and the quantum Yang-Baxter equation. Sov. Math. Dokl. 32, 254 (1985)

34. V.G. Drinfeld, Quasi Hopf algebras. Algebra Anal. 1, 6 (114) (1989). (Leningrad Math. J. 1:6, 1419 (1990))

35. T. Jurić, S. Meljanac, D. Pikutić, Realizations of $\kappa$-Minkowski space, Drinfeld twists and related symmetry algebras. Eur. Phys. J. C 75(5281506), 04955 (2015)

36. S. Meljanac, D. Meljanac, F. Mercati, D. Pikutić, Noncommutative spaces and Poincaré symmetry. Phys. Lett. B 766, 181-185 (2017). arXiv:1610.06716 [hep-th]

37. S. Meljanac, M. Stojic, New realizations of Lie algebra kappadeformed Euclidean space. Eur. Phys. J. C47, 531-539 (2006). arXiv:hep-th/0605133

38. S. Krešić-Jurić, S. Meljanac, M. Stojić, Covariant realizations of kappa-deformed space. Eur. Phys. J. C51, 229-240 (2007). arXiv:hep-th/0702215

39. S. Meljanac, S. Krešić-Jurić, Differential structure on kappaMinkowski space, and kappa-Poincare algebra. Int. J. Mod. Phys. A 26(20), 3385-3402 (2011). arXiv:1004.4647 
40. N. Loret, S. Meljanac, F. Mercati, D. Pikutić, Vector-like deformations of Minkowski space and relativistic kinematics to appear in Int. J. Modern Phys. D arXiv:1610.08310 [hep-th]

41. H.S. Snyder, Quantized space-time. Phys. Rev. 71(1), 38 (1947)

42. M.V. Battisti, S. Meljanac, Scalar field theory on noncommutative snyder space-time. Phys. Rev. D 82, 024028 (2010). arXiv: 1003.2108 [hep-th]

43. S. Meljanac, D. Meljanac, S. Mignemi, R. Štrajn, Snydertype spaces, twisted Poincaré algebra and addition of momenta. arXiv:1608.06207 [hep-th]

44. S. Meljanac, D. Meljanac, A. Samsarov, M. Stojić, Kappadeformed Snyder spacetime. Mod. Phys. Lett. A 25, 579-590 (2010). arXiv:0912.5087 [hep-th]

45. S. Meljanac, D. Meljanac, A. Samsarov, M. Stojić, Kappa Snyder deformations of Minkowski spacetime, realizations and Hopf algebra. Phys. Rev. D 83, 065009 (2011). arXiv:1102.1655 [hep-th]

46. T.R. Govindarajan, K.S. Gupta, E. Harikumar, S. Meljanac, D. Meljanac, Twisted statistics in kappa-Minkowski spacetime. Phys. Rev. D 77, 105010 (2008). arXiv:0802.1576 [hep-th]

47. T. Jurić, S. Meljanac, R. Štrajn, Twists, realizations and Hopf algebroid structure of kappa-deformed phase space. Int. J. Mod. Phys. A 29(5), 1450022 (2014). arXiv:1305.3088 [hep-th]

48. T. Jurić, S. Meljanac, R. Štrajn, $\kappa$-Poincaré-Hopf algebra and Hopf algebroid structure of phase space from twist. Phys. Lett. A 377 , 2472-2476 (2013). arXiv:1303.0994 [hep-th]

49. T. Jurić, D. Kovačević, S. Meljanac, $\kappa$-Deformed Phase Space, Hopf Algebroid and Twisting SIGMA 10, 106, p. 18 (2014). arXiv:1402.0397 [hep-th]

50. J. Lukierski, Z. Škoda, M. Woronowicz, Deformed covariant quantum phase spaces as Hopf algebroids. Phys. Lett. B 750, 401-406 (2015). arXiv:1507.02612 [hep-th]

51. S. Meljanac, Z. Škoda, M. Stojić, Lie algebra type noncommutative phase spaces are Hopf algebroids. Lett. Math. Phys. 107(3), 475503 (2017). arXiv:1409.8188

52. S. Meljanac, Z. Škoda, Hopf algebroid twists for deformation quantization of linear Poisson structures. arXiv:1605.01376 [hep-th]

53. S. Meljanac, A. Samsarov, R. Štrajn, Kappa-deformation of phase space; generalized Poincare algebras and R-matrix. JHEP 08, 127 (2012). arXiv:1204.4324 [hep-th]

54. T. Jurić, S. Meljanac, R. Štrajn, Universal $\kappa$-Poincaré covariant differential calculus over $\kappa$-Minkowski space. Int. J. Mod. Phys. A 29, 1450121 (2014). arXiv:1312.2751 [hep-th]
55. T. Juric, S. Meljanac, D. Pikutić, R. Štrajn, Toward the classification of differential calculi on $\kappa$-Minkowski space and related field theories. JHEP 1507, 055 (2015). arXiv:1502.02972 [hep-th]

56. A. Borowiec, A. Pachol, $\kappa$-Minkowski spacetime as the result of Jordanian twist deformation. Phys. Rev. D 79, 045012 (2009). arXiv:0812.0576 [hep-th]

57. S. Meljanac, D. Meljanac, A. Pachoł, D. Pikutić, Remarks on simple interpolation between Jordanian twists J. Phys. A 50(26), 265201 (2017). arXiv:1612.07984 [hep-th]

58. F. Girelli, E.R. Livine, Scalar field theory in Snyder space-time: alternatives. JHEP 1103, 132 (2011). arXiv:1004.0621 [hep-th]

59. S. Meljanac, D. Meljanac, S. Mignemi, R. Štrajn, Quantum field theory in generalised Snyder spaces. Phys. Lett. B 768, 321-325 (2017). arXiv:1701.05862 [hep-th]

60. S. Meljanac, S. Mignemi, J. Trampetić, J. You, Nonassociative Snyder $\phi^{4}$ quantum field theory. Phys. Rev. D 96, 045021 (2017). arXiv:1703.10851 [hep-th]

61. P. Aschieri, C. Blohmann, M. Dimitrijević, F. Meyer, P. Schupp, J. Wess, A gravity theory on non-commutative spaces. Class. Quant. Gravit. 22, 3511 (2005). arXiv:hep-th/0504183

62. P. Aschieri, M. Dimitrijević, F. Meyer, J. Wess, Noncommutative geometry and gravity Class. Quant. Gravit. 23, 1883 (2006). arXiv:hep-th/0510059

63. D. Meljanac, S. Meljanac, D. Pikutić, K.S. Gupta, Twisted statistics in Lie-deformed Minkowski spaces. arXiv:1703.09511 [hep-th]

64. E. Beggs, R. Makki, The Majid-Ruegg model and the Planck scales. arXiv:1306.4518 [hep-th]

65. E. Beggs, S. Majid, Gravity induced from quantum space time. Class. Quant. Gravit. 31, 035020 (2014). arXiv:1305.2403 [hepth]

66. A. Borowiec, T. Jurić, S. Meljanac, A. Pachoł, Central tetrads and quantum spacetimes. Int. J. Geom. Methods Mod. Phys. 13(08), 1640005 (2016). arXiv:1602.01292 [hep-th]

67. S. Majid, W. Tao, Noncommutative differentials on poisson-lie groups and pre-Lie algebras. arXiv:1412.2284 [hep-th]

68. S. Majid, W. Tao, Cosmological constant from quantum spacetime. arXiv:1412.2285 [hep-th] 